\title{
Braid Group, Gauge Invariance and Topological Order
}

\author{
Masatoshi Satd* and Mahito Kohmoto \\ Institute for Solid State Physics, The University of Tokyo, \\ Kashiwanoha 5-1-5, Kashiwa, Chiba, 277-8581, Japan \\ Yong-Shi $\mathrm{Wu}$ \\ Department of Physics, University of Utah, Salt Lake City, Utah 84112
}

(Dated: June 24, 2021)

\begin{abstract}
Topological order in two-dimensional systems is studied by combining the braid group formalism with a gauge invariance analysis. We show that flux insertions (or large gauge transformations) pertinent to the toroidal topology induce automorphisms of the braid group, giving rise to a unified algebraic structure that characterizes the ground-state subspace and fractionally charged, anyonic quasiparticles. Minimal ground state degeneracy is derived without assuming any relation between quasiparticle charge and statistics. We also point out that noncommutativity between large gauge transformations is essential for the topological order in the fractional quantum Hall effect.
\end{abstract}

PACS numbers: 05.30.Pr, 71.10.Hf, 71.10.Pm, 73.43.-f

In contrast to the expectation from classical thermodynamics, physics at absolute zero temperature is very rich because of novel effects of quantum fluctuations. In recent years it has become increasingly clear that in a wide class of two-dimensional strongly correlated many-body systems, transition driven by a non-thermal parameter may occur at zero temperature to a novel phase which can not be described by usual spontaneous symmetry breaking and order parameters. The characteristic signature of the novel phase is a finite ground state degeneracy that depends on the topology of the system; accompanying with it are charge fractionalization (with respect to that of the constituent particles) and/or fractional statistics of the quasiparticles. The first known example is the Laughlin state [1] for the fractional quantum Hall (FQH) effect, with electron filling factor $\nu=1 / n$ with $n$ odd. Soon after, it was realized that in this phase the ground state is $n$-fold degenerate on a cylinder [2] or on a torus [3], while is known nondegenerate on a sphere. Actually it is the ground state degeneracy that is responsible for the fractional quantization of Hall conductance [2, 4] and dictates the fractional charge $e *=e / n[5]$ and the anyon statistics $\theta=\pi / n[3,[6]$ of the quasiparticles. This type of new order is dubbed as topological order [7]. In recent years more systems, including bosonic ones or at zero magnetic field, are identified to possess topological order $[8,19,10,11,12,13,14,15,16,17]$.

In the study of topological order, a central issue is how to characterize or classify topological orders. Previously there has been the idea [2, 5] that the topology dependent ground state degeneracy seems to be dictated by an (emergent) discrete symmetry. But the latter was never identified explicitly, except being $Z_{n}$ for the Laughlin states. Another important issue is how to understand the relationship between ground state degen-

*Electronic address: msato@issp.u-tokyo.ac.jp eracy and charge fractionalization and/or quasiparticle statistics. A puzzling fact is that different patterns have appeared in investigations of various systems. For example, it was concluded [3] for the FQH systems that on a surface with non-zero genus $g$, the appearance of a fractional $\theta=\pi m / n$ statistics, with $m$ and $n$ co-primes, requires $n^{g}$-fold degenerate ground states, confirmed by the braid group analysis [6, 18] and by the effective ChernSimons theory as well [19, 20]. On the other hand, in a recent paper [21] it was shown, by using a gauge invariance argument [5], that charge fractionalization with $e^{*}=e p / q$, with $p$ and $q$ co-primes, requires a ground state degeneracy $q^{2 g}$ if the quasiparticles are ordinary bosons and fermions, while the FQH ground state degeneracy is known to be only $q^{g}$-fold [3, 18].

In this letter, we start with a reexamination of the interplay between charge fractionalization and quasiparticle anyon statistics, if they coexist, in constraining ground state degeneracy. This has been studied only for the Laughlin states and their variants 3, 18], where fractional charge and anyon statistics are known to be closely related to each other [22]. Below we shall derive minimal ground state degeneracy without assuming any relation between quasiparticle charge and statistics. A bonus of our reexamination is the identification of the discrete topological symmetry algebra that underlies the ground state degeneracy, which can be used to classify topological orders that support Abelian anyonic quasiparticle excitations.

We will start with the braid group formalism [6, 23, 24] for fractional statistics. Consider for $N$ quasiparticles in a toroidal system with size $L_{x} \times L_{y}$. The braid group generators [25] consist of $\sigma_{i}(i=1, \cdots, N-1)$, which exchanges the $i$ th and $(i+1)$ th particles clockwise without enclosing any other quasiparticle, and of $\tau_{i}$ and $\rho_{i}$ $(i=1, \cdots, N)$, which represent moving the $i$-th quasiparticle along a loop on the torus in $x$ - and $y$-direction, respectively. (See Fig 1.) Define operators $A_{i, j}$ and $C_{i, j}$ 
as

$$
A_{j, i}=\tau_{j}^{-1} \rho_{i} \tau_{j} \rho_{i}^{-1}, \quad C_{j, i}=\rho_{j}^{-1} \tau_{i} \rho_{j} \tau_{i}^{-1},
$$

where $1 \leq i<j \leq N$. The exchange operators $\sigma_{i}$ satisfy the following relations,

$$
\begin{aligned}
& \sigma_{k} \sigma_{l}=\sigma_{l} \sigma_{k}, \quad 1 \leq k \leq N-3, \quad|l-k| \geq 2 \\
& \sigma_{k} \sigma_{k+1} \sigma_{k}=\sigma_{k+1} \sigma_{k} \sigma_{k+1}, \quad 1 \leq k \leq N-2 \\
& \tau_{i+1}=\sigma_{i}^{-1} \tau_{i} \sigma_{i}^{-1}, \quad \rho_{i+1}=\sigma_{i} \rho_{i} \sigma_{i} \\
& \tau_{1} \sigma_{j}=\sigma_{j} \tau_{1}, \quad \rho_{1} \sigma_{j}=\sigma_{j} \rho_{1}, \quad \sigma_{i}^{2}=A_{i+1, i}
\end{aligned}
$$

where $1 \leq i \leq N-1$ and $2 \leq j \leq N-1$. And $\tau_{i}$ 's and $\rho_{i}$ 's satisfy

$$
\begin{aligned}
& A_{m, l} \tau_{k}=\tau_{k} A_{m, l}, \quad A_{m, l} \rho_{k}=\rho_{k} A_{m, l} \\
& \tau_{i} \tau_{j}=\tau_{j} \tau_{i}, \quad \rho_{i} \rho_{j}=\rho_{j} \rho_{i}, \\
& C_{j, i}=\left(\tau_{i} \tau_{j}\right) A_{j, i}^{-1}\left(\tau_{j}^{-1} \tau_{i}^{-1}\right), \quad A_{j, i}=\left(\rho_{i} \rho_{j}\right) C_{j, i}^{-1}\left(\rho_{j}^{-1} \rho_{i}^{-1}\right), \\
& C_{j, i}=\left(A_{j, j-1}^{-1} \cdots A_{j, i+1}^{-1}\right) A_{j, i}^{-1}\left(A_{j, i+1} \cdots A_{j, j-1}\right), \\
& \tau_{1} \rho_{1} \tau_{1}^{-1} \rho_{1}^{-1}=A_{2,1} A_{3,1} \cdots A_{N-1,1} A_{N, 1},
\end{aligned}
$$

where $1 \leq k<l<m \leq N$ and $1 \leq i<j \leq N$. a)

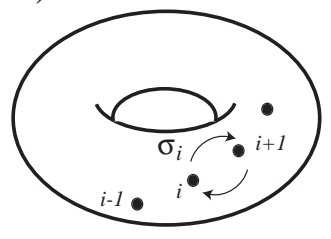

b)

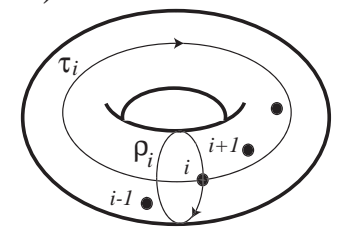

FIG. 1: a) An exchange of quasiparticles; b) Translations along non-shrinkable loops on the torus.

Let us now assume that the quasiparticles have a fractional charge $e^{*}=(p / q) e$, where $p$ and $q$ are mutually prime integers, and consider an adiabatic insertion of flux $2 \pi / e$ through one of the holes of the torus. If the adiabatic flux insertion induces an infinitesimal electric field in $x$-direction, the process can be realized by a large gauge transformation $U_{x}$, in which the $x$-component of the gauge field changes from $A_{x}=0$ to $A_{x}=2 \pi / e L_{x}$. After the large gauge transformation, the gauge potential $A_{x}=2 \pi / e L_{x}$ will give rise to an Aharanov-Bohm phase $e^{-2 \pi i p / q}$ when we apply $\tau_{i}$. Therefore, we obtain

$$
U_{x} \tau_{i}=e^{-2 \pi i p / q} \tau_{i} U_{x}
$$

On the other hand, because $\sigma_{i}$ and $\rho_{i}$ do not encircle the flux, we have

$$
U_{x} \rho_{i}=\rho_{i} U_{x}, \quad U_{x} \sigma_{i}=\sigma_{i} U_{x} .
$$

Similarly using the adiabatic flux insertion, we can define another large gauge transformation $U_{y}$, in which the $y$ component of the gauge potential changes from $A_{y}=0$ to $A_{y}=2 \pi / e L_{y}$; and we have

$$
U_{y} \tau_{i}=\tau_{i} U_{y}, \quad U_{y} \rho_{i}=e^{-2 \pi i p / q} \rho_{i} U_{y}, \quad U_{y} \sigma_{i}=\sigma_{i} U_{y} .
$$

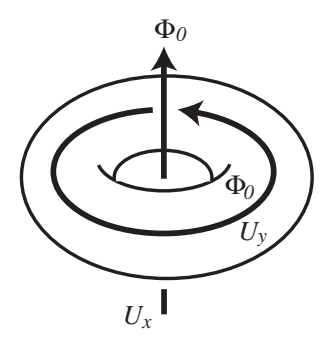

FIG. 2: Two possible insertions of a unit flux $\Phi_{0}$.

We notice that the relations (44), (5) and (6) are compatible with the braid group algebra (2) and (3). Thus the large gauge transformations $U_{a}(a=x, y)$ are (outer) automorphism of the braid group operators:

$$
\sigma_{i}^{\prime}=U_{a} \sigma_{i} U_{a}^{-1}, \quad \tau_{i}^{\prime}=U_{a} \tau_{i} U_{a}^{-1}, \quad \rho_{i}^{\prime}=U_{a} \rho_{i} U_{a}^{-1} .
$$

Namely by using relations (4), (5) and (6), one can check that the new operators $\sigma_{i}^{\prime}, \tau_{i}^{\prime}$ and $\rho_{i}^{\prime}$ also satisfy the same braid group algebra as $\sigma_{i}, \tau_{i}$ and $\rho_{i}$.

It is easy to verify that $U_{x} U_{y} U_{x}^{-1} U_{y}^{-1}$ commutes with all $\sigma_{i}, \tau_{i}$ and $\rho_{i}$. Therefore, by Schur's lemma, for any irreducible representation, $U_{x} U_{y} U_{x}^{-1} U_{y}^{-1}$ is a (unimodular) c-number; namely

$$
U_{x} U_{y}=e^{2 \pi i \lambda} U_{y} U_{x} .
$$

As we will see, $\lambda$ is rational and can be fixed by the requirement of a finite minimal ground state degeneracy. It is a new many-body quantum number, also characterizing the topological order of the system, and is closely related to the fractional quantization of Hall conductance (see below).

Assume that the quasiparticles are Abelian anyons:

$$
\sigma_{j}=e^{i \theta} \mathbf{1},
$$

where $\mathbf{1}$ is the unit matrix. Then the braid group representation is uniquely determined as

$$
\tau_{j}=e^{-2 i \theta(j-1)} T_{x}, \quad \rho_{j}=e^{2 i \theta(j-1)} T_{y},
$$

with $T_{x}$ and $T_{y}$ matrices satisfying

$$
T_{x} T_{y}=e^{-2 i \theta} T_{y} T_{x} .
$$

On a torus we also have the constraint on $N$ and $\theta$

$$
e^{2 i N \theta}=1 .
$$

Assuming $N \geq 2, \theta / \pi$ must be a rational number, $\theta=$ $\pi m / n$, where $m$ and $n$ are mutually prime integers. Thus, $T_{x}$ and $T_{y}$ satisfy

$$
T_{x} T_{y}=e^{-2 \pi i m / n} T_{y} T_{x} .
$$


The linear automorphisms induced by $U_{x}$ and $U_{y}$ now reduces to

$$
\begin{aligned}
& U_{x} T_{x} U_{x}^{-1}=e^{-2 \pi i p / q} T_{x}, \quad U_{y} T_{x} U_{y}^{-1}=T_{x}, \\
& U_{x} T_{y} U_{x}^{-1}=T_{y}, \quad U_{y} T_{y} U_{y}^{-1}=e^{-2 \pi i p / q} T_{y} .
\end{aligned}
$$

To count the ground state degeneracy, we consider the following process. First create $N$ pairs of quasiparticle and quasiholes out of the ground state, then move the $i$-th quasiparticle by $\tau_{i}$. After it returns to the original position, we pair annihilate all quasiparticles and quasiholes. This process defines an operation of $\tau_{i}$ to the ground states. Similarly, we define the operation of $\rho_{i}$ and $\sigma_{i}$ to the ground states. Throughout this letter, we assume that Fermi level lies in a gap and the gap remains finite in the operations above. Since for a system with Abelian anyonic excitations, the operations $\sigma_{i}$ 's on the ground state generate merely a phase. Thus we concentrate on the operations $\tau_{1}=T_{x}$ and $\rho_{1}=T_{y}$ on the ground state; from Eq. (10) all other $\tau_{i}$ and $\rho_{i}$ can be expressed in terms of them.

First, we reproduce the degeneracy due to the fractional statistics [6, 25]. Let us take the basis of the ground state to be an eigenstate of $T_{x}$,

$$
T_{x}|\eta\rangle=e^{i \eta}|\eta\rangle .
$$

By applying $T_{y}$ to $|\eta\rangle$ and using (13), the following new states are obtained:

$$
T_{x}\left(T_{y}^{s}|\eta\rangle\right)=e^{i(\eta-2 \pi s m / n)} T_{y}^{s}|\eta\rangle,
$$

where $s$ is an integer. Since the new states have $n$ different eigenvalues of $T_{x}$, the ground state has $n$-fold degeneracy at least.

The charge fractionalization gives another constraint for the degeneracy. From Eq.(13), $T_{x}$ and $T_{y}^{n}$ commute with each other,

$$
T_{x} T_{y}^{n}=T_{y}^{n} T_{x} .
$$

Therefore, we can take the basis of the ground states which diagonalize $T_{x}$ and $T_{y}^{n}$ simultaneously,

$$
T_{x}\left|\eta_{1}, \eta_{2}\right\rangle=e^{i \eta_{1}}\left|\eta_{1}, \eta_{2}\right\rangle, \quad T_{y}^{n}\left|\eta_{1}, \eta_{2}\right\rangle=e^{i \eta_{2}}\left|\eta_{1}, \eta_{2}\right\rangle .
$$

By applying $U_{x}$ and $U_{y}$ to this and using Eq.(14), we have

$$
\begin{aligned}
& T_{x}\left(U_{x}^{s} U_{y}^{t}\left|\eta_{1}, \eta_{2}\right\rangle\right)=e^{i\left(\eta_{1}+2 \pi s p / q\right)} U_{x}^{s} U_{y}^{t}\left|\eta_{1}, \eta_{2}\right\rangle \\
& T_{y}^{n}\left(U_{x}^{s} U_{y}^{t}\left|\eta_{1}, \eta_{2}\right\rangle\right)=e^{i\left(\eta_{2}+2 \pi t n p / q\right)} U_{x}^{s} U_{y}^{t}\left|\eta_{1}, \eta_{2}\right\rangle
\end{aligned}
$$

where $s$ and $t$ are integers. If $n / q=\mathcal{N} / \mathcal{Q}$ where $\mathcal{N}$ and $\mathcal{Q}$ are mutually prime integers, it is found that there are $q \mathcal{Q}$ sets of eigenvalues of $T_{x}$ and $T_{y}^{n}$. This implies that the ground state has $q \mathcal{Q}$-fold degeneracy at least.

By combining the results above, we find that the minimal degeneracy of the ground state should be the least common multiplet of $n$ and $q \mathcal{Q}=n \mathcal{Q}^{2} / \mathcal{N}$. Namely, the system has $n \mathcal{Q}^{2}$-fold ground state degeneracy. This indicates clearly that the fractional statistics and the charge fractionalization are both responsible for the ground state degeneracy. The minimal degeneracy obtained here includes both the results in Ref.[3] and Ref.[21] as special cases. More possibilities are predicted.

Up to now, we have not used non-commutating relation (8) between $U_{x}$ and $U_{y}$. This relation contains an additional parameter $\lambda$, which is not fixed uniquely by $e^{*}$ and $\theta$. We believe this parameter $\lambda$ could be determined by the low-energy effective Lagrangian, which we do not discuss here. In order for the degeneracy to be finite, $\lambda$ has to be a rational number $\lambda=k / l$, where $k$ and $l$ are co-primes. At least in the following examples we find that the integers $k$ and $l$ can be determined by requiring the degeneracy be minimal given $e^{*}$ and $\theta$. In any case, the degeneracy is given by a multiple of $n \mathcal{Q}^{2}$.

Now we present some explicit representations of $T_{x}$, $T_{y}, U_{x}$ and $U_{y}$ and corresponding degeneracy.

(1) $\theta=\pi / n$ and $e^{*}=e / n$. - This corresponds to the Laughlin state with $\nu=1 / n$. Because $\mathcal{N}=\mathcal{Q}=1$, the minimum degeneracy is $n$. If we assume that $U_{x}$ and $U_{y}$ satisfy

$$
U_{x} U_{y}=e^{-2 \pi i / n} U_{y} U_{x},
$$

we can construct $T_{x}, T_{y}, U_{x}$ and $U_{y}$ without increasing the degeneracy,

$$
\begin{aligned}
& T_{x}=S_{n \times n}, \quad T_{y}=R_{n \times n}, \\
& U_{x}=R_{n \times n}^{-1}, \quad U_{y}=S_{n \times n} .
\end{aligned}
$$

Here $S_{n \times n}=\operatorname{diag}\left\{1, e^{i 2 \pi / n}, \cdots, e^{i 2 \pi(n-1) / n}\right\}$ and

$$
R_{n \times n}=\left(\begin{array}{ccccc}
0 & 1 & 0 & \cdots & 0 \\
\vdots & 0 & \ddots & \ddots & \vdots \\
\vdots & \vdots & \ddots & \ddots & 0 \\
0 & \cdots & \cdots & 0 & 1 \\
1 & 0 & \cdots & \cdots & 0
\end{array}\right)
$$

They satisfy $S_{n \times n} R_{n \times n}=e^{-2 \pi i / n} R_{n \times n} S_{n \times n}$. This result reproduces the degeneracy given in Ref. [3].

(2) $\theta=0$ or $\theta=\pi$. - The quasiparticles are bosons or fermions. Since $n=1$, we obtain $\mathcal{N}=1$ and $\mathcal{Q}=q$. Thus the minimal degeneracy is $q^{2}$ [21]. We find that if $U_{x}$ and $U_{y}$ commutes with each other, the minimal degeneracy is realized as

$$
\begin{array}{ll}
T_{x}=R_{q \times q} \otimes 1_{q \times q}, & T_{y}=1_{q \times q} \otimes R_{q \times q}, \\
U_{x}=S_{q \times q}^{p} \otimes 1_{q \times q}, & U_{y}=1_{q \times q} \otimes S_{q \times q}^{p} .
\end{array}
$$

(3) $q$ and $n$ are mutually prime. - The degeneracy is $n q^{2}$. We can construct the following representation for $T_{x}, T_{y}, U_{x}$ and $U_{y}$ :

$$
\begin{aligned}
& T_{x}=1_{q \times q} \otimes R_{q \times q} \otimes S_{n \times n}^{m}, \\
& T_{y}=R_{q \times q} \otimes 1_{q \times q} \otimes R_{n \times n}, \\
& U_{x}=1_{q \times q} \otimes S_{q \times q}^{p} \otimes 1_{n \times n}, \\
& U_{y}=S_{q \times q}^{p} \otimes 1_{q \times q} \otimes 1_{n \times n},
\end{aligned}
$$


where the minimal degeneracy is realized. $U_{x}$ and $U_{y}$ commute with each other in this representation.

(4) $n=\mathcal{N} q$ and $m=1$. - Because of $\mathcal{Q}=1$, the minimum degeneracy is $n$. A representation is given by

$$
\begin{aligned}
& T_{x}=S_{n \times n}, \quad T_{y}=R_{n \times n}, \\
& U_{x}=R_{n \times n}^{-\mathcal{N} p}, \quad U_{y}=S_{n \times n}^{\mathcal{N} p} .
\end{aligned}
$$

$U_{x}$ and $U_{y}$ satisfy $U_{x} U_{y}=e^{-2 \pi i\left(\mathcal{N} p^{2} / q\right)} U_{y} U_{x}$.

(5) $q=\mathcal{Q} n$. - In this case, $\mathcal{N}=1$, thus the least degeneracy is $n \mathcal{Q}^{2}$. When $\mathcal{Q}$ and $n$ are mutually prime and $p=m=1$, we can construct the following representation:

$$
\begin{aligned}
& T_{x}=S_{\mathcal{Q} \times \mathcal{Q}} \otimes S_{n \times n} \otimes 1_{\mathcal{Q} \times \mathcal{Q}}, \\
& T_{y}=1_{\mathcal{Q} \times \mathcal{Q}} \otimes R_{n \times n} \otimes S_{\mathcal{Q} \times \mathcal{Q}}, \\
& U_{x}=R_{\mathcal{Q} \times \mathcal{Q}}^{-l} \otimes R_{n \times n}^{-k} \otimes 1_{\mathcal{Q} \times \mathcal{Q}}, \\
& U_{y}=1_{\mathcal{Q} \times \mathcal{Q}} \otimes S_{n \times n}^{k} \otimes R_{\mathcal{Q} \times \mathcal{Q}}^{-l},
\end{aligned}
$$

where $k / n+l / \mathcal{Q}=1 / \mathcal{Q} n(\bmod .1)$. In this case, $U_{x} U_{y}=$ $e^{-2 \pi i k^{2} / n} U_{y} U_{x}$.

Here we would like to mention that the noncommutativity of the large gauge transformation $U_{x} U_{y}=$ $e^{2 \pi i k / l} U_{y} U_{x}$ is closely related to the topological order in the fractional quantum Hall effect. To see this, consider the degenerate ground states $\phi_{K}(K=1, \cdots, d)$, on a torus with boundary conditions parametrized by twisted phases $\theta$ and $\varphi$ [4], satisfying $U_{x}|\theta, \varphi\rangle_{K}=|\theta+2 \pi, \varphi\rangle_{K}$ and $U_{y}|\theta, \varphi\rangle_{K}=|\theta, \varphi+2 \pi\rangle_{K}$. The Hall conductance is

$$
\frac{e^{2}}{h d} \sum_{K=1}^{d} \int_{0}^{2 \pi} \int_{0}^{2 \pi} \frac{d \theta d \varphi}{2 \pi i}\left[\left\langle\frac{\partial \phi_{K}}{\partial \varphi} \mid \frac{\partial \phi_{K}}{\partial \theta}\right\rangle-(\theta \leftrightarrow \varphi)\right] .
$$

Because of $U_{x}^{l} U_{y}=U_{y} U_{x}^{l}$, we can take the basis which diagonalizes both $U_{x}^{l}$ and $U_{y}$. In this basis a change in $\theta$ by $2 \pi l$ or in $\varphi$ by $2 \pi$ leads the state back to itself. Therefore, we have a torus with $0 \leq \theta<2 \pi l$ and $0 \leq$ $\varphi<2 \pi$. The above integral can be recast into

$\frac{e^{2}}{h d} \sum_{r=0}^{d / l-1} \int_{0}^{2 \pi l} \int_{0}^{2 \pi} \frac{d \theta d \varphi}{2 \pi i}\left[\left\langle\frac{\partial \phi_{r l+1}}{\partial \varphi} \mid \frac{\partial \phi_{r l+1}}{\partial \theta}\right\rangle-(\theta \leftrightarrow \varphi)\right]$, since the degenerate ground states $\phi_{K}$ satisfy $\phi_{r l+m}(\theta+$ $2 \pi, \varphi)=\phi_{r l+m+1}(\theta, \varphi)(r=0, \cdots, d / l-1, m=1, \cdots l-$ $1)$. Therefore we have the following formula for the Hall conductance, generalizing the result of Ref.[4]:

$$
\sigma_{x y}=\frac{e^{2}}{h d} \sum_{r=0}^{d / l-1} I_{r}=\frac{e^{2}}{h} \frac{I}{l} .
$$

Here $I_{r}$ is a generalized TKNN integer [26] defined by $\phi_{r l+1}$. For the second equality, we have noted that all $I_{r}$ 's take the same value $I$, since the degenerate ground states are related to each other by symmetry operations $T_{x}$ and $T_{y}$. This indicates clearly that the noncommutativity $\lambda=k / l$ of the large gauge transformations is essential to fractional quantization of the Hall conductance.

The fractional charge we discussed above can be any conserved $U(1)$ quantum number, with the flux threading understood as twisted boundary conditions. The minimal degeneracy obtained can be generalized to a highgenus Riemann surface: If the genus is $g$, for Abelian topological orders we find $g$ commuting copies of the discrete algebra presented above, so the minimal degeneracy is $n^{g} \mathcal{Q}^{2 g}$.

To conclude, in this letter we have proposed a discrete symmetry algebra, Eqs.(8), (13) and (14), of the operations $T_{x}, T_{y}, U_{x}$ and $U_{y}$, that completely characterizes the ground state subspace of a generic Abelian topological order, i.e. that supports Abelian anyonic excitations, on a torus. The identification verifies the old idea that the ground state degeneracy in a topological phase is due to the emergence of a discrete symmetry [2, 5]. We note that the algebra identified is indeed of topological origin and contains only three fractional parameters: quasiparticle charge $e^{*} / e$, anyon statistics $\theta / 2 \pi$ and flux noncommutativity $\lambda$. Ground state degeneracy is determined by the representations of this symmetry algebra.

Acknowledgement The work was supported in part by the U.S. NSF through grant No. PHY-0407187 (YSW). This work was begun in summer 2005, when YSW visited the ISSP, University of Tokyo. He thanks the financial support and warm hospitality from the host institution.
[1] R. B. Laughlin, Phys. Rev. Lett. 50, 1395 (1983).

[2] R. Tao and Y.-S. Wu, Phys. Rev. B30, 1097 (1984).

[3] X. G. Wen and Q. Niu, Phys. Rev. B41, 9377 (1990).

[4] Q. N. Niu, D. J. Thouless, and Y.-S. Wu, Phys. Rev. B31, 3372 (1985).

[5] Y. S. Wu, Y. Hatsugai, and M. Kohmoto, Phys. Rev. Lett. 66, 659 (1991).

[6] T. Einarsson, Phys. Rev. Lett. 64, 1995 (1990).

[7] X. G. Wen, Int. J. Mod. Phys. B4, 239 (1990).

[8] X. G. Wen, Phys. Rev. B44, 2664 (1991).

[9] X. G. Wen and A. Zee, Phys. Rev. B44, 274 (1991).

[10] N. Read and S. Sachdev, Phys. Rev. Lett. 66, 1773
(1991)

[11] T. Senthil and M. P. A. Fisher, Phys. Rev. B62, 7580 (2000).

[12] R. Moessner and S. L. Sondhi, Phys. Rev. Lett. 86, 1881 (2001).

[13] G. Misguich, D. Serban, and V. Pasquir, Phys. Rev. Lett. 89, 137202 (2002).

[14] L. Balents, M. P. A. Fisher, and S. M. Girvin, Phys. Rev. B65, 224412 (2002).

[15] O. I. Motrunich and T. Senthil, Phys. Rev. Lett. 89, 277004 (2002).

[16] M. Freedman, C. Nayak, K. Shthegel, K. Walker, and 
Z. Wang, Ann. Phys. 310, 428 (2004).

[17] M. C. Diamantini, P. Sodano, and C. A. Trugenberger, J. Phys. A39, L253 (2006).

[18] Y. Hatsugai, M. Kohmoto, and Y.-S. Wu, Phys. Rev. B43, 10761 (1991).

[19] X. G. Wen, Phys. Rev. B40, 7387 (1989).

[20] X. G. Wen, E. Dagotto, and E. Fradkin, Phys. Rev. B42, 6110 (1990).

[21] M. Oshikawa and T. Senthil, Phys. Rev. Lett. 96, 060601 (2006).
[22] J. R. S. D. Arovas and F. Wilczek, Phys. Rev. Lett. 53, 722 (1984).

[23] Y.-S. Wu, Phys. Rev. Lett. 52, 2103 (1984).

[24] Y. Hatsugai, M. Kohmoto, and Y.-S. Wu, Phys. Rev. B43, 2661 (1991).

[25] J. S. Birman, Comm. Pure Appl. Math. 22, 41 (1969).

[26] D. J. Thouless, M. Kohmoto, M. P. Nightingale, and M. den Nijs, Phys. Rev. Lett. 49, 405 (1982). 